\title{
CORRECTIONS
}

\section{Obituary: Syed Ahsanuddin Haider}

In this obituary by Brigid Hayden, we gave the wrong date of death for Dr Syed Ahsanuddin Haider (BMJ 2011;343:d4231, doi:10.1136/bmj.d4231). The correct date of death is 3 January
2011 [not 9 January 2001, as was published]. We apologise for this error.

Cite this as: $B M J 2011 ; 343: \mathrm{d} 4490$ 